\title{
INFLUÊNCIA DA QUANTIDADE DE VANÁDIO E POTÁSSIO ADICIONADA SOBRE ALUMINA DE TRANSIÇÃO
}

\author{
M. S. P. MARTINS ${ }^{1}$, J. A. J. RODRIGUES ${ }^{2}$ e G. G. CORTEZ ${ }^{1}$ \\ ${ }^{1}$ Escola de Engenharia de Lorena - USP - Departamento de Engenharia Química \\ ${ }^{2}$ Instituto Nacional de Pesquisas Espaciais-LCP \\ E-mail para contato: cortez@dequi.eel.usp.br
}

\begin{abstract}
RESUMO - Aluminas ativas são materiais importantes em inúmeras reações catalíticas, podem atuar como catalisador ou como suporte catalítico para metais. Através da reação de decomposição do isopropanol notou-se que o aumento na adição de vanádio diminuiu a atividade catalítica e o aumento da quantidade de vanádio e potássio na co-impregnação aumentou a taxa específica de reação.
\end{abstract}

\section{INTRODUÇÃO}

Aluminas são materiais estruturais importantes, com a ampla aplicação técnica. Particulamente, a $\gamma$-alumina que apresenta grande área de superfície, estrutura porosa e acidez superficial, é um importante suporte catalítico. (Ma, Zhu, 2009, Wang et al., 2009).

Óxidos de vanádio têm grande variedade de aplicações catalíticas (Cortez et al., 2003). O potássio é usualmente mencionado como promotor de catalisadores industriais (KiwiMinsker et al., 2001). A adição de $\mathrm{K}$ ao suporte de alumina impregnado com vanádio diminui a atividade, mas aumenta significativamente a seletividade do propeno (Ermini et al., 2000).

Neste estudo objetivou-se sintetizar um hidróxido de alumínio e calciná-lo para utilizálo como suporte; preparar catalisadores com diferentes quantidades de vanádio e potássio; caracterizar os suportes e catalisadores através das técnicas de volumetria de $\mathrm{N}_{2}$, redução à temperatura programada (RTP) e difratometria de raios $\mathrm{X}(\mathrm{DRX})$ e avaliar as propriedades ácidas e/ou básicas do catalisador através da reação de decomposição do isopropanol.

\section{EXPERIMENTAL}

\subsection{Síntese dos catalisadores}

Sintetizou-se o precursor através do método de precipitação com o uso de uma solução de $\mathrm{AlCl}_{3}$ e uma solução de $\mathrm{NaOH}$. Adicionou-se as duas soluções em um reator batelada sob agitação mecânica. Realizou-se a lavagem da amostra e então a deixou por uma noite na estufa. Esse material foi dividido em duas partes, ambas as partes foram calcinadas a $450{ }^{\circ} \mathrm{C}$. A primeira parte foi calcinada à taxa de $1,0{ }^{\circ} \mathrm{C} / \mathrm{min}$ e foi identificada como $\mathrm{Al}_{2} \mathrm{O}_{3}-\mathrm{I}$ (série I). A segunda parte foi calcinada à taxa de $3,34{ }^{\circ} \mathrm{C} / \mathrm{min}$ e recebeu o nome de $\mathrm{Al}_{2} \mathrm{O}_{3}$-II (série II). Em seguida, prepararam-se dois catalisadores de cada série. $\mathrm{Na}$ primeira série o primeiro catalisador foi preparado impregnando-se os suportes com 0,5 átomo de vanádio/ $/ \mathrm{nm}^{2}$ de alumina. A mistura da solução aquosa de $\mathrm{NH}_{4} \mathrm{VO}_{3}$ e o suporte foi realizada em um roto- 
evaporador a vácuo. Após a etapa de impregnação, o pó residual foi seco em estufa e depois calcinado a $450{ }^{\circ} \mathrm{C}$, obtendo-se assim o que se chamo de V/Al-I. Para se obter o segundo catalisador da série I, o suporte foi co-impregnado com uma quantidade total de 1 átomo $(\mathrm{V}+\mathrm{K})$ por $\mathrm{nm}^{2}$ de alumina. Para isso, utilizou-se soluções aquosas de $\mathrm{KOH}$ e $\mathrm{NH}_{4} \mathrm{VO}_{3}$ e, pelo mesmo método descrito acima, obteve-se o catalisador identificado por VK/Al-I. Na série II os catalisadores foram preparados pelo mesmo método descrito acima, entretanto o primeiro catalisador foi preparado impregnando-se os suportes com 4 átomos de vanádio $/ \mathrm{nm}^{2}$ de alumina (V/Al-II) e o segundo catalisador foi impregnado com uma quantidade total de 8 átomos por $\mathrm{nm}^{2}$ de alumina (VK/Al-II). Os catalisadores foram caracterizados pelas técnicas de volumetria de $\mathrm{N}_{2}$, DRX e RTP e pela reação de decomposição do isopropanol.

\subsection{Caracterização físico-química}

As medidas de áreas específicas foram determinadas a partir das isotermas de adsorçãodessorção de $\mathrm{N}_{2}$ a $-196{ }^{\circ} \mathrm{C}$, usando-se o equipamento Quantachrome, modelo NOVA 1000 para a série I e Belsorp II-mini para a série II.

As análises de DRX foram realizadas utilizando-se o método do pó, através do equipamento da marca Panalytical, modelo Enpyrean. As fases cristalinas foram identificadas através do programa graph analyzer criado pelo INPE de Cachoeira Paulista.

As análises de RTP dos catalisadores suportados foram realizadas em um equipamento Quantachrome, modelo Chembet-3000, equipado com um detector de condutividade térmica.

As propriedades ácidas e/ou básicas dos catalisadores foram avaliadas através da reação de decomposição do isopropanol. O reator foi alimentado com isopropanol que foi injetado na tubulação do reator com o auxílio de uma bomba (Thermo Separation Products, modelo P100) e diluído em hélio. Os gases efluentes do reator foram analisados por cromatografia gasosa, utilizando-se um cromatógrafo da marca Varian, modelo 3380, equipado com um detector de condutividade térmica (DCT), e conectado em linha com o sistema reacional através de uma válvula de injeção automática. Para separação e análise dos efluentes do reator foi utilizada uma coluna de aço inox empacotada com fase estacionária Poropak-Q $(4,5 \mathrm{~m})$. A reação foi avaliada um tempo de residência $\left(\mathrm{W} / \mathrm{F}_{\mathrm{A} 0}\right)$ igual a $6,3 \mathrm{~g} . \mathrm{h} / \mathrm{mol}$. A taxa específica de formação dos produtos (TEP) foi calculada a partir das equações abaixo:

$$
\begin{array}{r}
\text { TEP }= \\
(1) \quad \frac{S_{P} \times T E R}{S_{B E T}} \\
\\
T E R=\frac{X_{A} \times F_{A 0}}{W \times S_{B E T}}
\end{array}
$$

$$
X_{A}(\%)=\left(\frac{n_{R}}{n_{R_{0}}}\right) \times 100
$$


$S_{P}(\%)=\left(\frac{n_{R}}{n_{R_{0}}}\right) \times\left(\frac{N_{C P}}{N_{C R}}\right) \times 100$,

onde TER é a taxa específica de reação, $\mathrm{X}_{\mathrm{A}}$ é a conversão do isopropanol, $\mathrm{S}_{\mathrm{P}} \mathrm{a}$ seletividade dos produtos, $n_{R}$ é o número de mols do isopropanol consumido, ${ }_{R} 0$ é o número de mols de isopropanol na alimentação e ${ }^{N_{C P}}$ e ${ }^{N_{C R}}$ são os números de átomos de carbono presentes nos produtos formados e no isopropanol, respectivamente.

\section{RESULTADOS E DISCUSSÕES}

As áreas específicas $\left(\mathrm{S}_{\mathrm{BET}}\right)$ e o volume total de poros $(\mathrm{Vp})$ são apresentados na Tabela 1. Comparando-se os suportes com os catalisadores, de ambas as séries, observou-se que a impregnação da alumina com vanádio e a co-impregnação com vanádio e potássio geraram uma diminuição no valor da área específica, uma vez que os poros foram ocupados pelas espécies de V e K. Ao se comparar os suportes e os catalisadores da série I com os da série II, observou-se que o aumento na quantidade de vanádio e potássio causou também uma redução na área específica. Por outro lado, observou-se uma pequena variação no volume de poros quando os catalisadores das duas séries foram comparados. Uma maior variação foi visualizada quando se comparou o suporte com os dois catalisadores da série II.

Tabela 1 - Valores de área específica $\left(\mathrm{S}_{\mathrm{BET}}\right)$ e volume de poros $(\mathrm{Vp})$.

\begin{tabular}{ccccc}
\hline Catalisadores & $\mathrm{V}_{2} \mathrm{O}_{5}(\% \mathrm{p} / \mathrm{p})$ & $\mathrm{K}_{2} \mathrm{O}(\% \mathrm{p} / \mathrm{p})$ & $\mathrm{S}_{\mathrm{g}}\left(\mathrm{m}^{2} / \mathrm{g}\right)$ & $\mathrm{Vp}\left(\mathrm{cm}^{3} / \mathrm{g}\right)$ \\
\hline $\mathrm{Al}_{2} \mathrm{O}_{3}-\mathrm{I}$ & - & - & 340 & 0,32 \\
$\mathrm{~V} / \mathrm{Al}-\mathrm{I}$ & 4,9 & - & 303 & 0,34 \\
$\mathrm{VK} / \mathrm{Al}-\mathrm{I}$ & 4,9 & 2,5 & 330 & 0,33 \\
\hline $\mathrm{Al}_{2} \mathrm{O}_{3}-\mathrm{II}$ & - & - & 263 & 0,37 \\
$\mathrm{~V} / \mathrm{Al}-\mathrm{II}$ & 24,1 & - & 243 & 0,30 \\
$\mathrm{VK} / \mathrm{Al}-\mathrm{II}$ & 24,1 & 2,0 & 239 & 0,29 \\
\hline
\end{tabular}

A Figura 1 mostra as isotermas de adsorção-dessorção de $\mathrm{N}_{2}$ a $-196{ }^{\circ} \mathrm{C}$. Para todas as amostras foram observadas isotermas do tipo IV, onde o ramo inferior mostra a quantidade de nitrogênio adsorvido com o aumento da pressão relativa, e o ramo superior representa a quantidade do gás dessorvido no processo inverso. Esse tipo de isoterma é característico de sólidos mesoporosos e macroporosos (Teixeira et al., 2001).

Figura 1- Isotermas de adsorção-dessorção dos suportes e catalisadores.
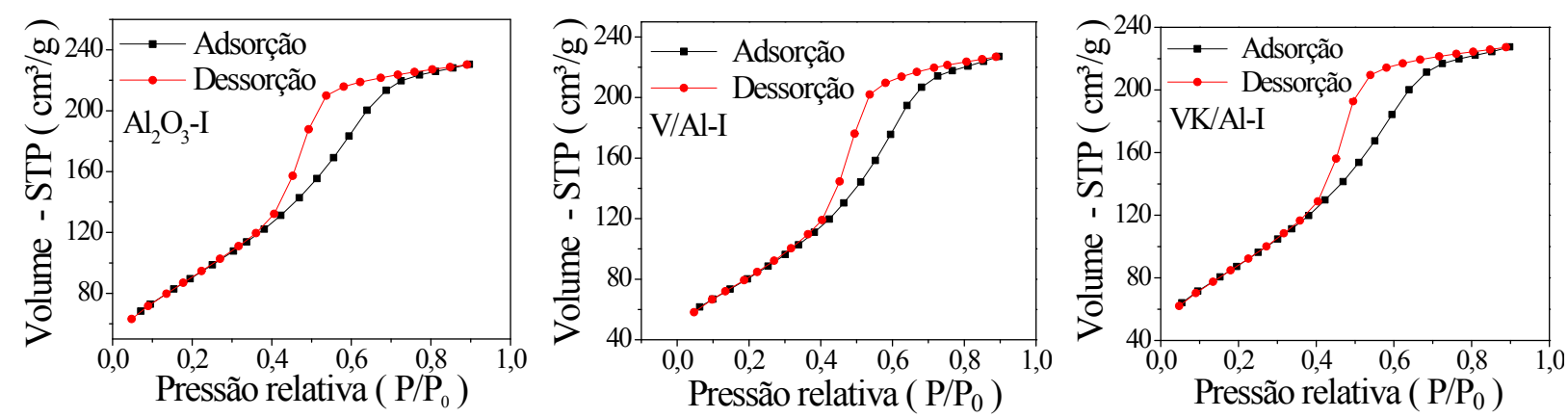

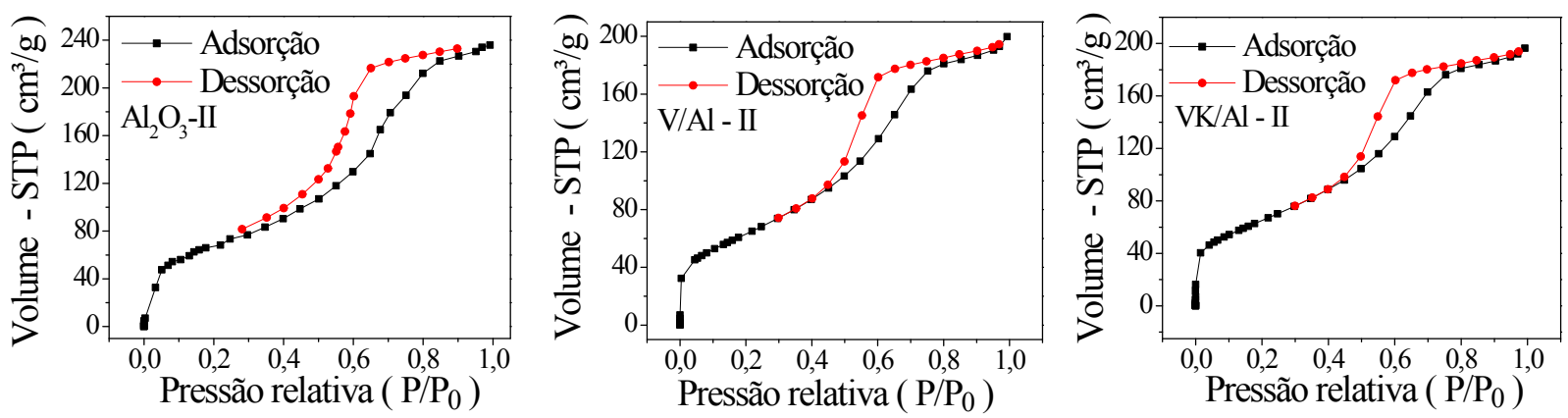

A Figura 2 mostra a distribuição do diâmetro de poros e do volume de poros. Os catalisadores e os suportes apresentaram um comportamento monomodal, com distribuição de poros na faixa de 30 a $70 \AA$ para os suportes e catalisadores da série I e de 0 a $10 \mathrm{~nm}$ para os da série II. O aumento inicial no volume de poros a baixas pressões relativas é devido a presença de microporos nos catalisadores.

Figura 2 - Volume de poros versus diâmetro de poros para os suportes e catalisadores.
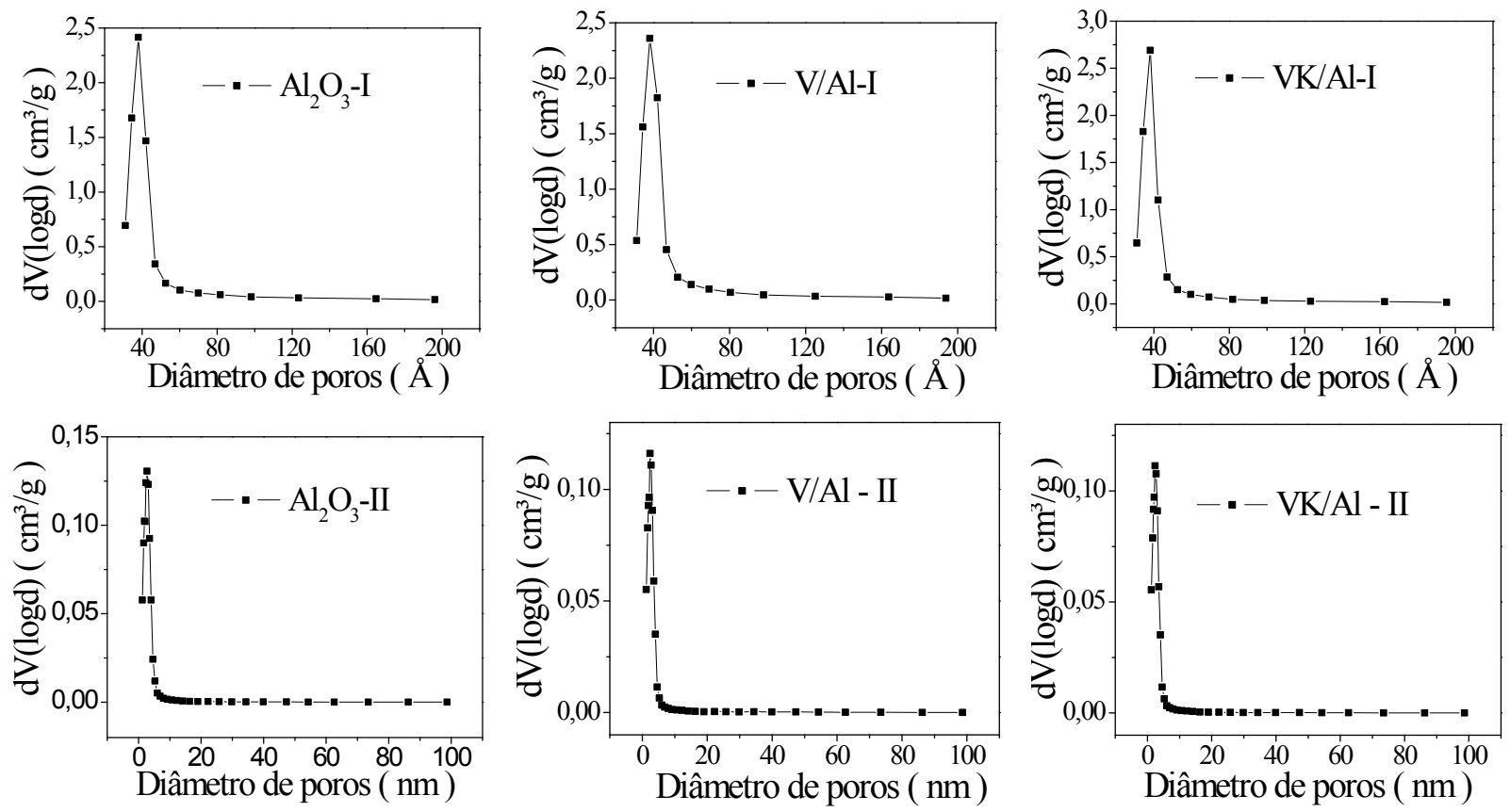

As análises de RTP dos catalisadores são apresentadas na Figura 3. O óxido de vanádio apresenta três temperaturas máximas de redução a 655,690 e $823{ }^{\circ} \mathrm{C}$, que corresponde a seguinte etapa de redução das espécies de vanádio, descrito por Korane et al. (1994):

$$
\mathrm{V}_{2} \mathrm{O}_{5} \rightarrow \mathrm{V}_{6} \mathrm{O}_{13}\left(675^{\circ} \mathrm{C}\right) ; \mathrm{V}_{6} \mathrm{O}_{13} \rightarrow \mathrm{V}_{2} \mathrm{O}_{4}\left(705^{\circ} \mathrm{C}\right) ; \mathrm{V}_{2} \mathrm{O}_{4} \rightarrow \mathrm{V}_{2} \mathrm{O}_{3}\left(780^{\circ} \mathrm{C}\right)
$$

Os catalisadores apresentaram um único pico de redução do vanádio, correspondente à redução de $\mathrm{V}_{2} \mathrm{O}_{5}$ a $\mathrm{V}_{6} \mathrm{O}_{13}$. Comparando-se os catalisadores $\mathrm{V} / \mathrm{Al}$ com os catalisadores $\mathrm{VK} / \mathrm{Al}$, notou-se que a co-impregnação com potássio causou um aumento na temperatura de redução do vanádio desde 574 a $588{ }^{\circ} \mathrm{C}$, nos catalisadores da série I e um aumento de 506 à $549{ }^{\circ} \mathrm{C}$, nos da série II. Em contrapartida, comparando-se entre si os catalisadores impregnados apenas com vanádio, notou-se que o aumento na quantidade de vanádio impregnado no suporte gerou 
uma redução na temperatura de redução do vanádio de 574 para $506{ }^{\circ} \mathrm{C}$. O mesmo ocorreu para os catalisadores co-impregnados com vanádio e potássio, notou-se que houve uma diminuição de 588 para $549{ }^{\circ} \mathrm{C}$. A diminuição da temperatura de redução do vanádio pode estar relacionada a uma interação entre o vanádio e o potássio sobre o suporte que estaria dificultando a redução do vanádio, assim quanto maior a quantidade de vanádio e potássio adicionada ao suporte, menor a temperatura de redução do vanádio. Cabe ressaltar que os picos de consumo de $\mathrm{H}_{2}$ apresentaram intensidades diferentes para as duas séries já que foram usadas quantidades diferentes de catalisadores no teste do TPR.

Figura 3 - RTP dos catalisadores

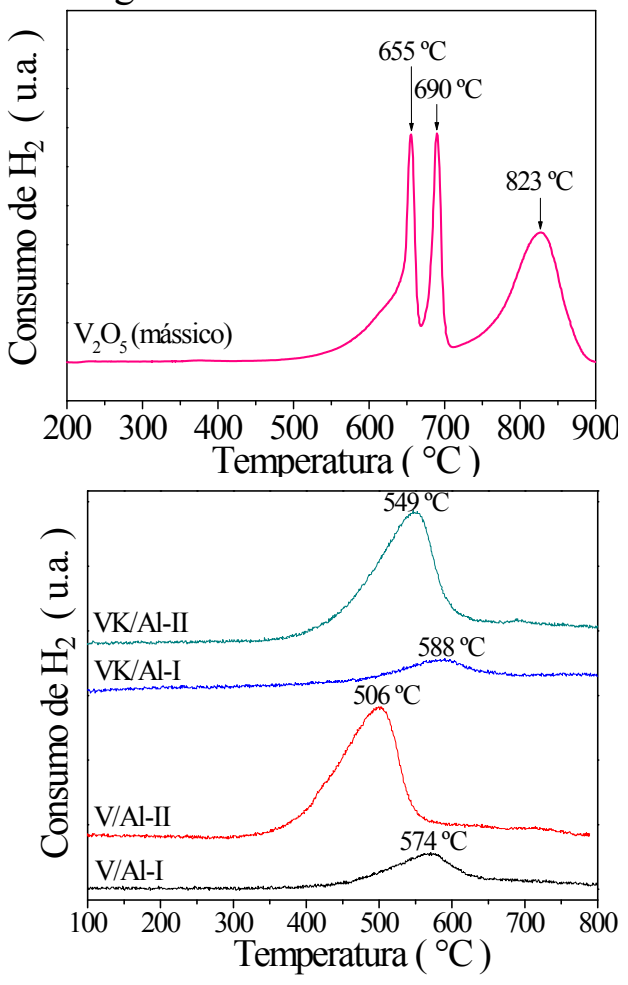

Figura 4 - DRX do precursor e das amostras calcinadas. Picos referentes às fases $(\bullet) \gamma-\mathrm{Al}_{2} \mathrm{O}_{3}$, $(\square) \eta-\mathrm{Al}_{2} \mathrm{O}_{3},(\bullet)$ boemita e ( $\left.\square\right)$ baierita.

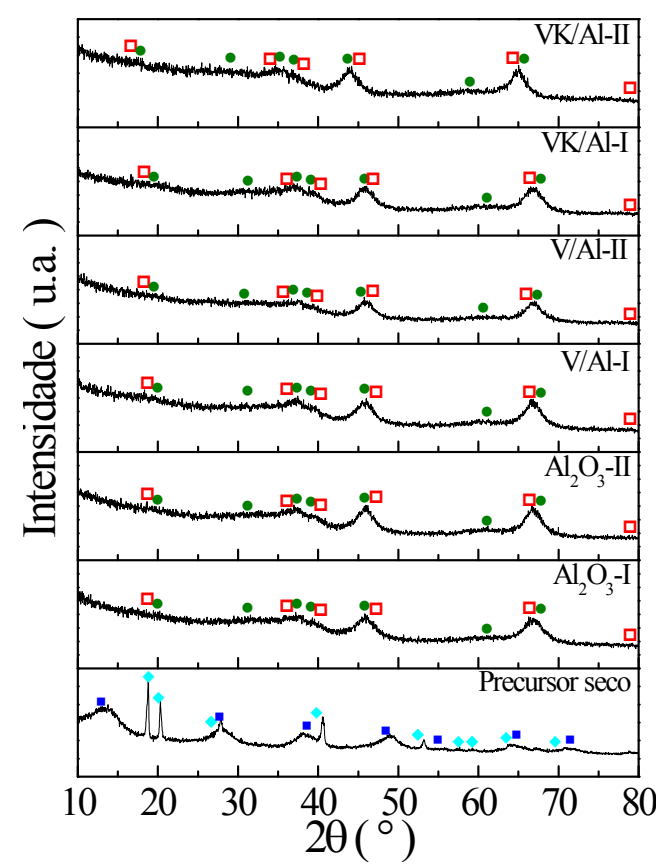

Os perfis de DRX dos suportes e catalisadores são apresentados na Figura 4. O precursor seco, preparado a partir do método da precipitação, apresentou as fases boemita, com estrutura ortorrômbica, e baierita, com estrutura monoclínica. Assim, como já era esperado, o DRX das amostras calcinadas comprovou a presença das aluminas de transição $\gamma$ $\mathrm{Al}_{2} \mathrm{O}_{3}$ e $\eta-\mathrm{Al}_{2} \mathrm{O}_{3}$, respectivamente. A impregnação com vanádio e a co-impregnação com vanádio e potássio não provocou mudanças significativas no DRX. A quantidade de vanádio e potássio impregnada no suporte não alterou as fases presentes nas duas séries. A ausência de picos referente ao vanádio e ao potássio nas amostras impregnadas indica que seus óxidos podem estar presentes em estado amorfo, ou altamente dispersos sobre o suporte.

Tabela 2 - Resultados da taxa específica de reação (TER) e da taxa específica de formação dos produtos (TEP) dos catalisadores a $250{ }^{\circ} \mathrm{C}$.

\begin{tabular}{cccc}
\hline \multirow{2}{*}{ Catalisadores } & TER & \multicolumn{2}{c}{ TEP $\left(\mu \mathrm{mol}^{-2} \cdot \mathrm{min}^{-1}\right) / 250{ }^{\circ} \mathrm{C}$} \\
\cline { 2 - 4 } & $\left(\mu \mathrm{mol} \cdot \mathrm{m}^{-2} \cdot \mathrm{min}^{-1}\right)$ & Propeno & Éter diisopropílico \\
\cline { 3 - 4 } $\mathrm{Al}_{2} \mathrm{O}_{3}$-I & 13,1 & 11,5 & 1,4 \\
$\mathrm{Al}_{2} \mathrm{O}_{3}$-II & 22,5 & 21,5 & 0,8 \\
\hline
\end{tabular}




\begin{tabular}{cccc}
\hline V/Al-I & 17,4 & 16,5 & 0,7 \\
V/Al-II & 10,3 & 8,8 & 1,5 \\
\hline VK/Al-I & 0,9 & 0,7 & 0,1 \\
VK/Al-II & 4,2 & 3,7 & 0,3 \\
\hline
\end{tabular}

Os resultados da atividade catalítica, obtidos na reação de decomposição do isopropanol, na temperatura de $250{ }^{\circ} \mathrm{C}$ são mostrados na Tabela 2. Para a série I, a impregnação do suporte com vanádio aumentou a taxa de reação e a produção de propeno e causou uma diminuição na produção de éter. Já na co-impregnação com vanádio e potássio observou-se uma diminuição na taxa de reação e na produção de propeno, e a produção de éter foi praticamente desprezível. Para a série II, a impregnação com vanádio e a coimpregnação com vanádio e potásssio reduziu a atividade catalítica e a produção de propeno. A produção de éter também diminuiu quando houve a co-impregnação, entretanto, na impregnação apenas com vanádio a quantidade de éter produzida aumentou. Quando as séries I e II foram comparadas, notou-se que o aumento na quantidade de vanádio adicionada sobre o suporte diminuiu a atividade catalítica. Entretanto, o aumento na adição de vanádio e potássio sobre a alumina, pelo método da co-impregnação, aumentou a atividade catalítica.

\section{CONCLUSÕES}

As propriedades texturais do suporte foram modificadas pela adição de vanádio e potássio, efeito esse atribuído ao bloqueio dos microporos por essas espécies. Assim, quanto maior a quantidade de vanádio e potássio impregnada menor o valor de área específica. A síntese do precursor revelou, no DRX, que a quantidade de $\mathrm{V}$ e $\mathrm{K}$ adicionada não alterou as fases presentes no material. O precursor apresentou as fases boehmita e baierita e os catalisadores calcinados apresentaram as fases $\gamma-\mathrm{Al}_{2} \mathrm{O}_{3}$ e $\eta-\mathrm{Al}_{2} \mathrm{O}_{3}$. Nos resultados do RTP, os catalisadores apresentaram um único pico de redução do vanádio que corresponde à redução de $\mathrm{V}_{2} \mathrm{O}_{5}$ a $\mathrm{V}_{6} \mathrm{O}_{13}$. $\mathrm{O}$ aumento na quantidade dos metais impregnados diminuiu a temperatura de redução do vanádio. A partir da reação de decomposição do isopropanol, conclui-se que o aumento na adição de vanádio ao suporte diminuiu a atividade catalítica. Já o aumento na adição de vanádio e potássio ao suporte através da co-impregnação aumentou a taxa específica de reação. Conclui-se também que os catalisadores apresentaram basicamente sítios ácidos, evidenciados pela formação de propeno e éter diisopropílico.

\section{REFERÊNCIAS}

CORTEZ, G.G.; FIERRO, J.L.G.; BAÑARES, M.A. Role of potassium on the structure and activity of alumina-supported vanadium oxide catalysts for propane oxidative dehydrogenation. Catalysis Today, v. 78, p. 219-228, 2003.

ERMINI, V.; FINOCCHIO E.; SECHI S.; BUSCA, G.; ROSSINI S., Propane oxydehydrogenation over alumina-supported vanadia doped with manganese and potassium. Appl. Catal., v. 198, p. 67-79, 2000.

KIWI-MINSKER, L.; BULUSHEV, D.A.; RAINONE, F.; RENKEN, A. Implication of the acid-base properties of V/Ti-oxide catalyst in toluene partial oxidation. Journal of Molecular Catalysis, v. 184, p. 223-235, 2002. 
MA, M.G.; ZHU, J.F. A facile solvothermal route to synthesis of $\gamma$-alumina with bundle-like and flower-like morphologies, Mater. Lett., v. 63, p. 881-883, 2009.

TEIXEIRA, V. G.; COUTINHO, F. M. B.; GOMES, A. S. Principais métodos de caracterização da porosidade de resinas à base de divinilbenzeno. Quim. Nova, v. 24(6), p. 808-818, 2001.

WANG, Y.; WANG, J.; SHEN, M.; WANG, W. Synthesis and properties of thermostable calumina prepared by hydrolysis of phosphide aluminum, J. Alloys Compd., v.467, p.405-412, 2009. 